$\begin{array}{ll}\text { Jennifer A. Ohtola, MD, PhD } & \text { Sandra J. Hong, MD } \\ \text { Fellow, Department of Allergy and Clinical Im- } & \text { Staff, Department of Allergy and Clinical Immunology, } \\ \text { munology, Respiratory Institute, Cleveland Clinic; } & \text { Respiratory Institute, Cleveland Clinic; Director, Food } \\ \text { Clinical Instructor, Cleveland Clinic Lerner College } & \begin{array}{l}\text { Allergy Center of Excellence, Cleveland Clinic; Clinical } \\ \text { of Medicine of Case Western Reserve University, }\end{array} \\ \begin{array}{l}\text { Associate Professor, Cleveland Clinic Lerner College } \\ \text { of Medicine of Case Western Reserve University, } \\ \text { Cleveland, } \mathrm{OH}\end{array} & \begin{array}{l}\text { Cleveland, } \mathrm{OH}\end{array}\end{array}$

\title{
BRIEF ANSWERS
}

TO SPECIFIC

CLINICAL

QUESTIONS

\section{Q: Does my patient need an allergy evaluation for penicillin allergy?}

\begin{abstract}
A: Maybe. A detailed clinical history should be obtained directly from patients to determine their risk of penicillin allergy. Those deemed at low risk may not require a formal allergy evaluation. The recently validated PEN-FAST penicillin allergy clinical-decision rule $^{1}$ may be useful for clinicians of all specialties to direct appropriate strategies and, for some patients, to potentially remove the "penicillin allergy" label from their medical record.
\end{abstract}

\section{HOW COMMON IS PENICILLIN ALLERGY?}

Beta-lactam antibiotics, which include the penicillins, are among the safest and most effective antibiotics and are widely used. However, $5 \%$ to $10 \%$ of the US population has reported allergies to penicillins, with a higher prevalence in older and hospitalized patients. ${ }^{2}$ Patients with a documented penicillin allergy are more likely to receive alternative broadspectrum antibiotics, which can lead to higher healthcare expenditures, longer hospital stays, higher risk of adverse events, and development of drug-resistant pathogens.

Despite the high frequency of documented allergy, more than $95 \%$ of hospitalized patients labeled as having penicillin allergy can actually tolerate this class of drug without adverse reactions. ${ }^{3}$ This discrepancy between reported and actual penicillin allergy may be explained by the waning of penicillin-specific immunoglobulin $\mathrm{E}$ ( $\mathrm{IgE}$ ) antibodies over time. It is estimated that up to $80 \%$ of patients with a history of immediate clinical symptoms compatible with a penicillin allergy become tolerant of it after a decade if there has been no further exposure. After 20 years, fewer than $1 \%$ of patients continue to maintain their sensitivity. ${ }^{2}$

doi:10.3949/ccjm.89a.21004
Other factors contributing to the discrepancy between reported and clinically relevant allergy may include confounding symptoms related to the patient's illness, misclassification of adverse reactions, or both. "Unknown" is a frequently documented type of reaction in electronic medical records, as are cutaneous reactions including rash and hives. ${ }^{3}$

Although most patients may be able to tolerate penicillins safely, keep in mind that penicillins are the most common cause of druginduced fatal and nonfatal anaphylaxis in the United States, particularly when they are given parenterally. ${ }^{4}$ As a general rule, IgE-mediated allergic reactions typically occur within minutes after receiving the drug and may present as anaphylaxis, urticaria, bronchospasm, angioedema, or hypotension. Penicillins have also been associated with other severe nonIgE-mediated reactions such as drug reaction with eosinophilia and systemic symptoms and Stevens-Johnson syndrome/toxic epidermal necrolysis.

\section{WHAT QUESTIONS SHOULD I ASK THE PATIENT?}

Components essential to an allergic history include how long ago the reaction occurred, symptom details, timing of symptom onset, duration of reaction, treatment of the reaction, and use of same or similar medication since. When relevant, concomitant medications, diagnoses, laboratory results, and imaging details should be reviewed.

While allergy specialists widely agree on these components of an allergic history, few decision tools are available for other clinicians to direct appropriate diagnosis and management of penicillin allergy. In addition, obtaining such a history may be difficult in many pa- 
tients who are unable to clearly recall the drug to which they reacted or details surrounding the reaction.

\section{HOW DO I STRATIFY A PATIENT'S RISK?}

After the allergy history is determined, patients can be stratified as being at low, moderate, or high risk for penicillin allergy to determine if skin testing or drug challenge or both are appropriate. To date, there is no universally accepted definition of risk levels. The need for the development of validated tools to support clinical decision-making has been recognized. However, efforts have been limited by the lack of generalizability across various populations and effective implementation strategies. $^{5}$

\section{The PEN-FAST decision rule}

PEN-FAST, a penicillin allergy decision rule, was recently developed to aid clinicians in point-of-care risk assessment. ${ }^{1}$ A prospective cohort of 622 penicillin allergy-tested patients from 2 primary Australian sites and 3 retrospective cohorts from Australia and the United States were subjected to internal and external validation, respectively. The following features associated with a positive penicillin test were used to create the mnemonic PEN-FAST:

- PENicillin allergy reported (proceed with the assessment below)

- Five years or less since a reaction, or unknown interval (2 points)

- Anaphylaxis or angioedema, or Severe cutaneous reaction ( 2 points)

- Treatment was required for the allergy episode (1 point). ${ }^{1}$

A score of 0 indicates a very low risk of a positive penicillin allergy test $(<1 \%)$, a score of 1 or 2 indicates a low risk (5\%), a score of 3 indicates a moderate risk (20\%), and a score of 4 or 5 indicates a high risk (50\%). A cutoff of less than 3 points was found to have a negative predictive value of $96.3 \% .^{1}$

PEN-FAST has been further validated in a large European cohort of adult patients reporting a reaction with amoxicillin. ${ }^{6}$ These studies suggest PEN-FAST may be an effective tool for non-allergy-trained clinicians to use in estimating risk in patients across various populations.

\section{WHAT TESTING IS AVAILABLE FOR EVALUATING PENICILLIN ALLERGY?}

Figure 1 outlines our approach for a patient with reported penicillin allergy, starting with risk stratification using PEN-FAST.

A direct or graded amoxicillin challenge under medical observation may be performed in low-risk patients, ie, a PEN-FAST score of 2 or less. ${ }^{3,78}$ Before performing such a procedure, it is essential to obtain informed consent and ensure appropriate monitoring and access to medications used to treat allergic reactions. If no reaction occurs during the observation period, the patient can subsequently take any type of penicillin without restriction. For those with reported allergy to penicillin only, any other beta-lactam (including cephalosporins, carbapenems, and monobactams) can be given as indicated. Drug challenge with the culprit penicillin (if not amoxicillin) is also a reasonable option.

Penicillin skin testing is recommended for patients at moderate or high risk (PENFAST score $\geq 3$ ) or with unknown history, current pregnancy, or hemodynamic instability. The skin test is the most reliable way to demonstrate penicillin-specific $\operatorname{IgE}$ antibodies. However, it does not predict the risk of non-IgE-mediated reactions or development of IgE-mediated allergic reactions upon future exposures to penicillins. For this reason, skin testing, drug challenge, and desensitization are not recommended in patients with a history of severe delayed reaction such as StevensJohnson syndrome-toxic epidermal necrolysis, drug reaction with eosinophilia and systemic symptoms, interstitial nephritis, serum sickness, or hemolytic anemia.

Penicillin skin testing is performed using both major and minor antigenic determinants in a stepwise evaluation with percutaneous followed by intradermal testing. Medical providers, including clinicians, advanced practice practitioners, and registered nurses, who have been adequately trained, can perform penicillin skin testing.

Patients who have a positive skin test result are assumed to be allergic to penicillin and should not undergo a penicillin challenge test. However, they can undergo desensitization to penicillin if they truly need it to induce a state
PEN-FAST may be an effective point-of-care decision tool for penicillin allergy 


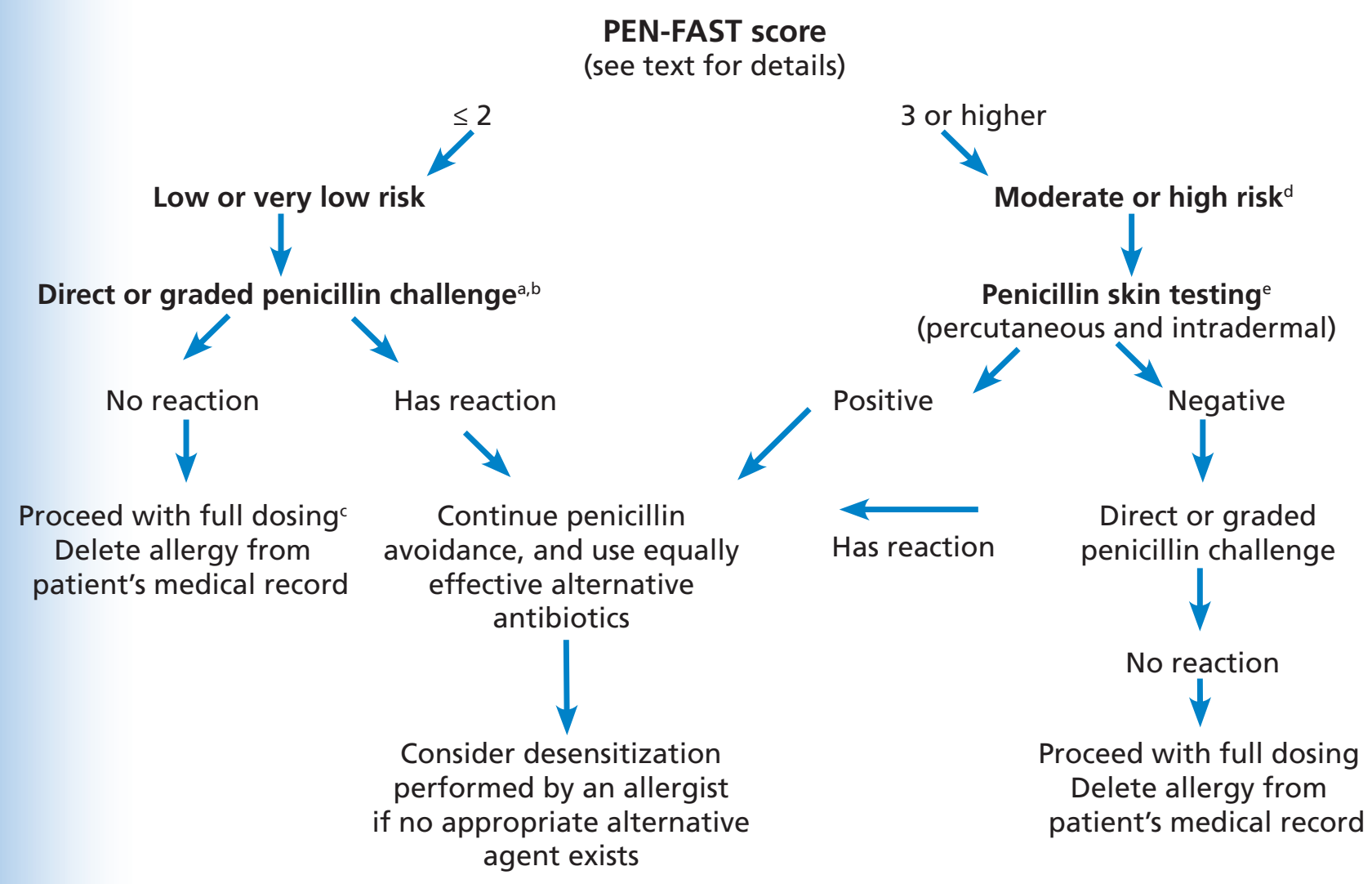

Figure 1. Recommended approach to patients with reported penicillin allergy based on PEN-FAST risk stratification. ${ }^{a} \mathrm{~A}$ drug challenge can be performed by a non-allergist or allergist under medical observation. Informed consent must be obtained from the patient.

${ }^{b}$ For a direct challenge in an adult patient, a single dose of $250 \mathrm{mg}$ of amoxicillin followed by 60 to 120 minutes of observation is a common approach. Graded amoxicillin challenges are often performed by giving one-tenth of the full dose followed by the remaining dose with 30 to 60 minutes of observation between steps. In pediatric patients, amoxicillin challenges are performed using weight-based dosing. Drug challenge with the culprit penicillin could also be considered.

'Any type of penicillin can be given without restriction. For those with reported allergy to penicillin only, any other beta-lactam can be utilized as indicated.

${ }^{d}$ Skin testing and drug challenge are contraindicated in patients with a history of severe delayed reaction such as Stevens-Johnson syndrome/toxic epidermal necrolysis, drug reaction with eosinophilia and systemic symptoms, interstitial nephritis, serum sickness, or hemolytic anemia attributed to penicillin use.

eSkin testing should be performed only by adequately trained providers.

PEN-FAST = PENicillin allergy reported; Five years or less since a reaction, or unknown interval; Anaphylaxis or angioedema, or Severe cutaneous reaction; Treatment was required for the allergy episode

of temporary tolerance. It is reasonable to repeat skin testing if many years have passed, due to the waning of penicillin-specific $\operatorname{IgE}$ antibodies.

Those with negative skin test results should proceed with a drug challenge as described in Figure 1. The predictive value of negative penicillin skin testing is approximately $95 \%$ and in combination with oral amoxicillin challenge approaches 100\%. ${ }^{3}$

Serum-specific IgE assays are available for a number of selected penicillins, but they have low sensitivity and thus have limited value and are not commonly used.

\section{THE BOTTOM LINE}

In patients with a reported penicillin allergy, obtaining a detailed allergic history directly from the patient is essential. Clinical-decision tools such as PEN-FAST may be useful to identify patients for whom the penicillin allergy label can be removed without a formal allergy evaluation, thus facilitating optimal antibiotic therapy and reducing drug costs.

\section{DISCLOSURES}

The authors report no relevant financial relationships which, in the context of their contributions, could be perceived as a potential conflict of interest. 


\section{OHTOLA AND HONG}

\section{REFERENCES}

1. Trubiano JA, Vogrin S, Chua KYL, et al. Development and validation of a penicillin allergy clinical decision rule. JAMA Intern Med 2020; 180(5):745-752. doi:10.1001/jamainternmed.2020.0403

2. Broyles AD, Banerji A, Barmettler S, et al. Practical guidance for the evaluation and management of drug hypersensitivity: specific drugs. J Allergy Clin Immunol Pract 2020; 8(9S):S16-S116. doi:10.1016/j.jaip.2020.08.006

3. Shenoy ES, Macy E, Rowe T, Blumenthal KG. Evaluation and management of penicillin allergy: a review. JAMA 2019; 321(2):188-199. doi:10.1001/jama.2018.19283

4. Castells M, Khan DA, Phillips EJ. Penicillin allergy. N Engl J Med 2019; 381(24):2338-2351. doi:10.1056/NEJMra1807761

5. Stone CA Jr, Trubiano J, Coleman DT, et al. The challenge of delabeling penicillin allergy. Allergy 2020; 75(2):273-288. doi:10.1111/all.13848
6. Piotin A, Godet J, Trubiano JA, et al. Predictive factors of amoxicillin immediate hypersensitivity and validation of PEN-FAST clinical decision rule. Ann Allergy Asthma Immunol 2021. doi:10.1016/j.anai.2021.07.005

7. Kuruvilla M, Shih J, Patel K, Scanlon N. Direct oral amoxicillin challenge without preliminary skin testing in adult patients with allergy and at low risk with reported penicillin allergy. Allergy Asthma Proc 2019; 40(1):57-61. doi:10.2500/aap.2019.40.4184

8. Cooper L, Harbour J, Sneddon J, Seaton RA. Safety and efficacy of de-labelling penicillin allergy in adults using direct oral challenge: a systematic review. JAC Antimicrob Resist 2021. doi:10.1093/jacamr/dlaa123

Address: Sandra J. Hong, MD, Department of Allergy and Clinical Immunology, Respiratory Institute, A90, Cleveland Clinic, 9500 Euclid Avenue, Cleveland, $\mathrm{OH}$ 44195; hongs3@ccf.org 